\title{
Assessing the Performance of Ultrafast Vector Flow Imaging in the Neonatal Heart via Multiphysics Modeling and In Vitro Experiments
}

\author{
Joris Van Cauwenberge, Student Member, IEEE, Lasse Lovstakken, Member, IEEE, \\ Solveig Fadnes, Associate Member, IEEE, Alfonso Rodriguez-Morales, \\ Jan Vierendeels, Patrick Segers, and Abigail Swillens
}

\begin{abstract}
Ultrafast vector flow imaging would benefit newborn patients with congenital heart disorders, but still requires thorough validation before translation to clinical practice. This paper investigates 2-D speckle tracking (ST) of intraventricular blood flow in neonates when transmitting diverging waves at ultrafast frame rate. Computational and in vitro studies enabled us to quantify the performance and identify artifacts related to the flow and the imaging sequence. First, synthetic ultrasound images of a neonate's left ventricular flow pattern were obtained with the ultrasound simulator Field II by propagating point scatterers according to 3-D intraventricular flow fields obtained with computational fluid dynamics (CFD). Noncompounded diverging waves (opening angle of $60^{\circ}$ ) were transmitted at a pulse repetition frequency of $9 \mathrm{kHz}$. ST of the B-mode data provided 2-D flow estimates at $180 \mathrm{~Hz}$, which were compared with the CFD flow field. We demonstrated that the diastolic inflow jet showed a strong bias in the lateral velocity estimates at the edges of the jet, as confirmed by additional in vitro tests on a jet flow phantom. Furthermore, ST performance was highly dependent on the cardiac phase with low flows $(<5 \mathrm{~cm} / \mathrm{s})$, high spatial flow gradients, and out-ofplane flow as deteriorating factors. Despite the observed artifacts, a good overall performance of 2-D ST was obtained with a median magnitude underestimation and angular deviation of, respectively, $28 \%$ and $13.5^{\circ}$ during systole and $16 \%$ and $10.5^{\circ}$ during diastole.
\end{abstract}

Index Terms-Blood flow measurement, medical imaging, medical signal and image processing.

\section{INTRODUCTION}

$\mathbf{K}$ NOWLEDGE of the full 3-D intracardiac blood flow pattern would enhance current visualization tools and quantitative assessment of important cardiac parameters, such

Manuscript received June 7, 2016; accepted June 30, 2016. Date of publication August 1, 2016; date of current version November 1, 2016. The computational resources (Stevin Supercomputer Infrastructure) and services used in this work were provided by the VSC (Flemish Supercomputer Center), funded by Ghent University, the Hercules Foundation and the Flemish Government - Department EWI. The work of A. Swillens was supported by a Post-Doctoral grant of the Research Foundation Flanders (FWO) under Grant 1276416N.

J. Van Cauwenberge, P. Segers, and A. Swillens are with the Institute of Biomedical Technology, Ghent University, Ghent 9000, Belgium (e-mail: abigail.swillens@ugent.be).

L. Lovstakken, S. Fadnes, and A. Rodriguez-Morales are with the Department of Circulation and Medical Imaging, NTNU, Trondheim 7491, Norway.

J. Vierendeels is with the Department of Flow, Heat and Combustion Mechanics, Ghent University, Ghent 9000, Belgium.

Digital Object Identifier 10.1109/TUFFC.2016.2596804 as volumetric flows (cardiac output, pulmonary blood flow, shunt flow, etc.) or pressure gradients [1], [2]. One particular patient population likely benefiting from such a proper quantification are newborns [3], since congenital heart disease (CHD) is diagnosed in about 4-8 in 1000 births [4], [5]. CHD like atrial or ventricular septal defect (VSD), patent ductus arteriosus, or more complex conditions like tetralogy of Fallot and hypoplastic left/right ventricles all imply significantly altered intracardiac blood flow patterns [2]. To assess the severity, pediatric cardiologists will first and foremost rely on echocardiography to: 1) visualize the anatomy of the heart and 2) inspect the cardiac flow [3]. Doppler-based imaging techniques are currently used in clinical blood flow analysis, such as spectral analysis techniques [Continuous Wave (CW) and Pulsed Wave (PW) Doppler] for local velocities and color-coded techniques Color Flow Imaging (CFI) for flows in a wider field of view. Unfortunately, these flow estimators have inherent limitations, which may lead to faulty diagnoses [6], [7]. First of all, only the velocity component parallel to the ultrasonic beam is captured, neglecting the intrinsic 3-D character of the hemodynamics. Considering the swirling motion and vortices [1], [8]-[10] commonly seen in cardiac flow (e.g., the left ventricular diastolic vortex), clearcut interpretation of such 1-D-measurements is not straightforward. In addition, both CFI and PW Doppler are troubled by aliasing, limiting the velocity range, and leading to even more ambiguous results. Clearly, improved multidimensional flow estimators are necessary.

To overcome the above limitations, several techniques have already been suggested and developed. Crossed-beam Doppler techniques [11], [12] apply trigonometry on several independent axial velocity estimates to obtain a vector flow estimation, while the transverse oscillation method estimates the lateral velocity component from a laterally oscillating pressure field [13], artificially synthesized in receive. Both techniques are hampered by aliasing artifacts, yet yield promising results for vascular applications [14]-[16]. However, with observed blood flow velocities up to $3 \mathrm{~m} / \mathrm{s}$ (e.g., VSD) in neonatal cardiac imaging, aliasing artifacts can be detrimental. This limitation is to some extent alleviated by speckle tracking (ST) [17], [18], a block matching technique to track speckle, the characteristic signature of the received echoes 
arising in the presence of randomly distributed scatterers (red blood cells) that cannot be individually discriminated due to insufficient spatial resolution. As such, speckle reflects the constructive and destructive interference patterns of the ultrasound waves backscattered on the particles. This speckle pattern remains relatively constant as the blood moves except in the presence of, e.g., strong blood velocity gradients or out-of-plane blood flow, decorrelating the speckle pattern and hampering the performance of pattern matching techniques. The maximal detectable velocity can be flexibly adapted by modifying the search region at the expense of increased computational times. ST has already been implemented on commercial scanners in 3-D, though for cardiac tissue motion detection [19]. However, ST for cardiac flow imaging is still in a research phase with studies previously presented in 2-D, both for neonatal [20] and for adult cardiac flow imaging [21], but also in 3-D [22]. The latter study did, however, presume the use of contrast agents, called echoparticle image velocimetry [23], [24], facilitating tracking but also requiring expensive and invasively administered contrast agent.

Beside these advances in multidimensional velocity estimation, the advent of high frame rate imaging has boosted the development of cardiac flow imaging [25], [26]. Acquiring ultrasonic data at a kilohertz $(\mathrm{kHz})$ rate is nowadays possible by transmitting plane [27], [28] or diverging waves [29], insonifying the whole region of interest at once, and receiving the ultrasonic echoes through parallel receive beamforming, speeding up the acquisition rate. The use of plane waves has been investigated for cardiac 1-D [30], [31] and 2-D flow imaging [32], [33], but resulted in a limited rectangular field of view, requiring several steered plane waves to cover an adequate field of view. Cone-shaped insonified regions are more appropriate for cardiac applications and can be accomplished by applying a virtual negative focus [29], resulting in an acoustic wave diverging with depth.

In vivo and in vitro demonstrations of diverging waves for cardiac tissue motion and blood velocity quantification have been presented in adults as well. Papadacci et al. [34] used spatial coherently compounded diverging waves to measure shear wave propagation in cardiac tissues, thereby estimating elasticity and tissue motion. High frame rate intraventricular color flow visualization was demonstrated by Osmanski et al. [35], while to the best of our knowledge, only Takahashi et al. [21], [36] provided a proof of concept for vector flow imaging using magnitude compounded diverging waves.

So far, a thorough quantitative analysis of the use of diverging waves for 2-D ST of the intraventricular flow patterns has been lacking though. Hence, this paper aims to quantitatively explore the performance of diverging waves for high frame rate vector flow imaging in the clinically relevant case of the neonatal heart. Note that the smaller dimensions of the newborn heart require higher frequency probes, which might additionally challenge the vector flow estimation compared with its adult counterpart when using diverging waves due to the tradeoff between penetration and resolution. We will use an inhouse developed multiphysics modeling approach [37], [38], creating virtual ultrasound data with the true hemodynamics behind the image fully known, allowing to validate the 2-D flow estimates toward a ground truth. This methodology relies on an ultrasonic imaging simulation approach, representing blood flow by moving point scatterers on which the ultrasound waves reflect. The movement of these point scatterers will be derived from a computational fluid dynamics (CFD) model of neonatal intraventricular flow. This multiphysics approach has previously demonstrated the opportunities and shortcomings of flow imaging in the carotid artery [39]-[41] and the vascular access site for hemodialysis [42]. The findings from our simulation environment will be further supported by high frame rate vector flow measurements on an in vitro jet flow phantom.

\section{Methods}

First, the subsequent steps of the multiphysics modeling approach as outlined in Fig. 1 will be presented before describing the in vitro experimental setup. Key component of the multiphysics modeling is the ultrasound simulator Field II [43], [44], which models blood flow as a set of moving point scatterers upon which the ultrasound waves reflect. To account for the complex 3-D intraventricular flow motion, the point scatterers will be propagated according to a time-varying flow field in a neonatal left ventricular cavity as computed with appropriate numerical techniques (CFD) [Fig. 1(a) and (b)]. Since the CFD velocity fields are directly used as input to the Field II simulator, they can be considered as the ground truth when evaluating the final synthetic ultrasound images. The scatterer positions are dynamically updated during the simulated ultrasonic scanning procedure, i.e., the emission of diverging waves and parallel receive beamforming to ensure radio frequency (RF) data acquisition in the $\mathrm{kHz}$ range [Fig. 1(c)]. Finally, postprocessing the RF data with a 2-D ST algorithm provides the estimated vector flow field [Fig. 1(d)], which is then compared with the ground-truth CFD velocity field [Fig. 1(a)], allowing us to quantitatively assess the ST performance and identification of possible artifacts.

\section{A. Computational Flow Phantom}

Using a commercial CFD solver (ANSYS Fluent), we calculated the time-varying intraventricular blood flow field in a generic model of a neonatal left ventricle with its temporal volumetric change mathematically described. The diastolic geometry created with ANSYS DesignModeler [Fig. 2(a)] consisted of a truncated prolate spheroid [45], [46], with a long-axis to short-axis ratio $(\alpha)$ fixed to 2 , and was topped with a circular outlet and ellipsoidal inlet representing, respectively, the aortic valve $(\varnothing 6.5 \mathrm{~mm})$ and the mitral valve (equivalent $\varnothing 8.0 \mathrm{~mm}$ ). The geometry was subsequently meshed with ANSYS ICEM CFD, resulting in a tetrahedral mesh of 378480 cells.

We imposed the volume change of this truncated prolate spheroid associated with systolic contraction or diastolic expansion $V(t)$ according to a previously reported 


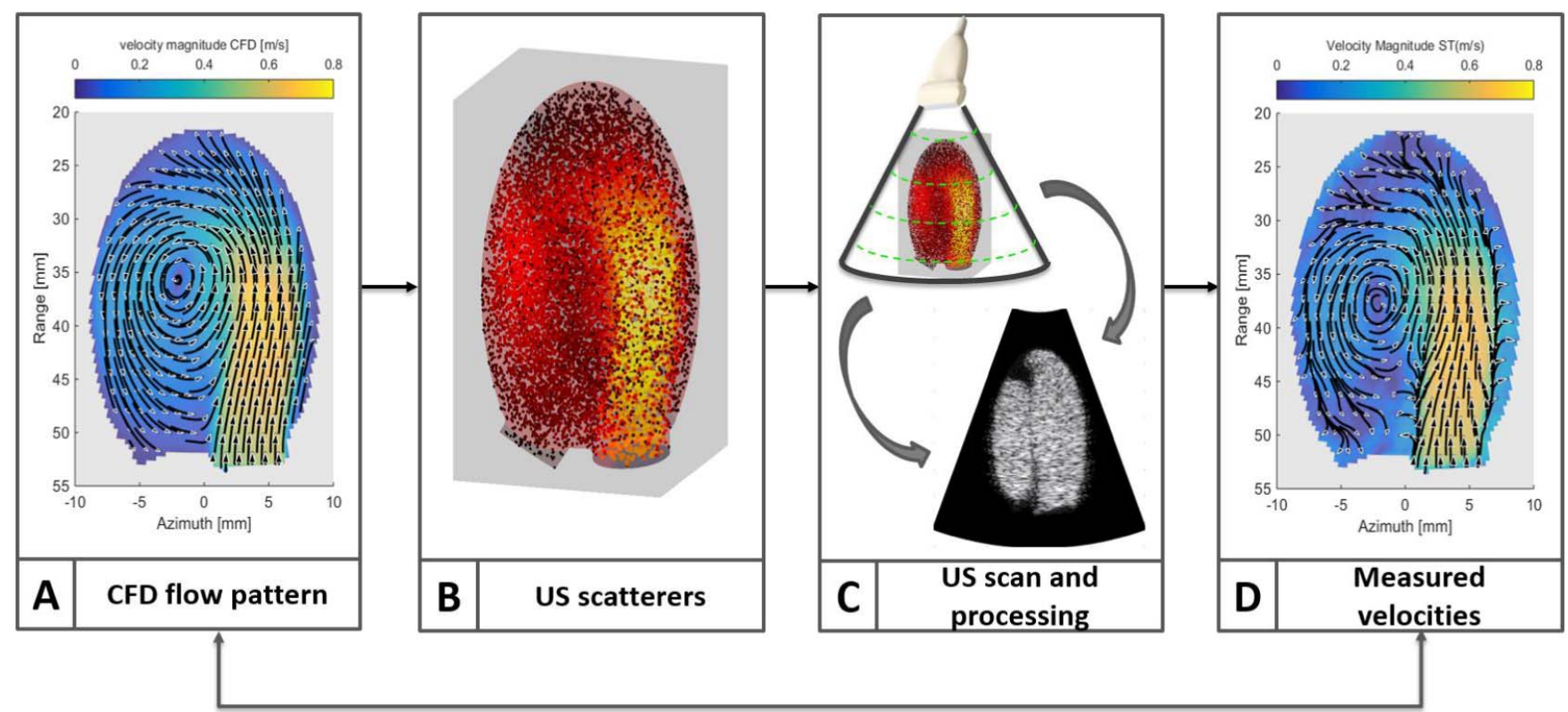

Fig. 1. Multiphysics modeling approach. In the domain of the (A) fluid dynamical model, generated with CFD, (B) ultrasonic scatterers are initiated and subsequently propagated during the (C) ultrasonic scanning acquisition according to the CFD velocities. From the resulting RF data, a (D) flow field can be estimated. Knowledge of the ground truth (A) allows validating the imaging and processing techniques quantitatively.

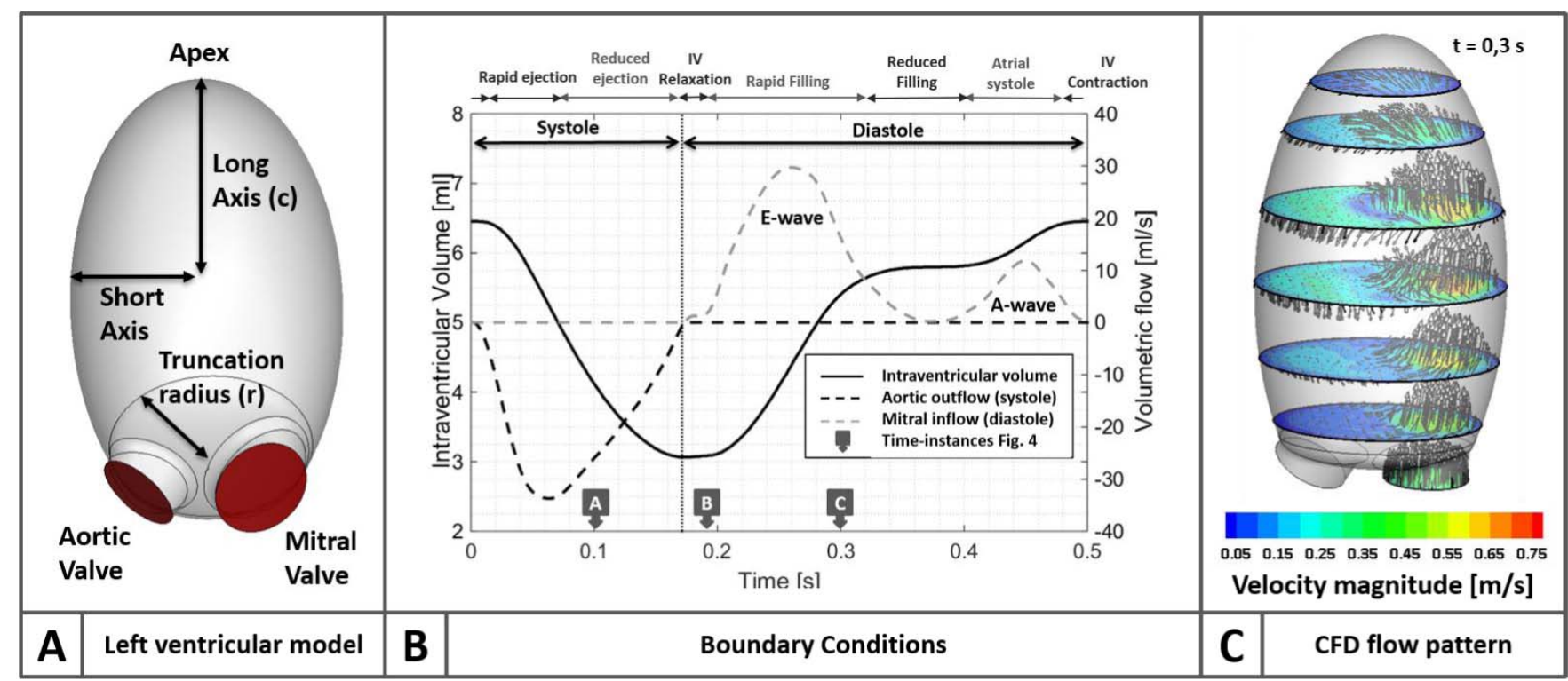

Fig. 2. (A) Simplified computational flow phantom representing the neonatal left ventricular cavity was created with the CAD program AN SYS DesignModeler. (B) Appropriate boundary conditions needed to be applied during both the systolic and diastolic phases indicated by, respectively, the black and gray dashed lines. (C) Time-varying 3-D flow field was obtained with a finite volume method in the commercial CFD solver ANSYS Fluent. The time instance shown in (C) represents the end of the rapid filling phase.

mathematical model [45]

$V(t)=\pi * \frac{c(t)^{3}}{\alpha^{2}}\left(\frac{2}{3}+\sqrt{1-\frac{\alpha^{2} * r^{2}}{c(t)^{2}}}+\frac{\left(-\sqrt{1-\frac{\alpha^{2} * r^{2}}{c(t)^{2}}}\right)^{3}}{3}\right)$

with $r$ the radius at the truncation of the spheroid, fixed at $7 \mathrm{~mm}$, and $c(t)$ the long-axis length of the prolate spheroid, both indicated in Fig. 2(a). This intraventricular volume change [the solid line in Fig. 2(b)-different cardiac phases indicated] resulted in physiologically realistic mitral and aortic flows [the dashed lines in Fig. 2(b)]. An end-diastolic volume (EDV) of $6.74 \mathrm{~mL}$ was chosen, which is in the physiological range [47]-[49]. Together with an ejection fraction (EF) of $52.5 \%$ [50], these choices led to an end-systolic volume of $3.2 \mathrm{~mL}$. The heart rate was set to 120 beats $/ \mathrm{min}$.

To account for this volumetric change, each node of the computational mesh needed to be displaced at each time step of the CFD simulation. A user-defined arbitrary 
TABLE I

ULTRASOUND PARAMETERS

\begin{tabular}{|c|c|c|c|}
\hline Parameter & Value (simulations/in-vitro) & Parameter & Value (simulations/in-vitro) \\
\hline Probe & $\begin{array}{l}\text { 1D phased array, neonatal cardiac imaging / } \\
1 \mathrm{D} \text { phased array, } \mathrm{P} 4-2 \mathrm{~V} \text {, adult cardiac imaging }\end{array}$ & PRF & $9 \mathrm{kHz} / 6 \mathrm{kHz}$ \\
\hline Center frequency (fo) & $6 \mathrm{MHz} / 2,98 \mathrm{MHz}$ & virtual source location & $-13.3 \mathrm{~mm} /-32,1 \mathrm{~mm}$ \\
\hline $\begin{array}{l}\text { Relative bandwidth } \\
\text { (BW) }\end{array}$ & $75 \% / 60 \%$ & transmitted pulses & $2 / 4$ \\
\hline $\begin{array}{l}\text { Transducer element } \\
\text { (lateral x elevation) }\end{array}$ & $96 \times 1 / 64 \times 1$ & $\begin{array}{l}\text { Sampling frequency RF } \\
\text { channel data }\end{array}$ & $100 \mathrm{MHz} / 11,90 \mathrm{MHz}$ \\
\hline Pitch & $0.15 \mathrm{~mm} / 0,5797 \mathrm{~mm}$ & Receive apodization & Hamming (dynamic aperture) \\
\hline Kerf & $0.03 \mathrm{~mm} / 0,097 \mathrm{~mm}$ & $\begin{array}{l}\text { Sampling frequency IQ } \\
\text { beamformed data }\end{array}$ & $48 \mathrm{MHz} / 5,95 \mathrm{MHz}$ \\
\hline $\begin{array}{l}\text { Height and width } \\
\text { transducer }\end{array}$ & $\begin{array}{l}\text { Height: } 10 \mathrm{~mm} / 30 \mathrm{~mm} \\
\text { Width: } 14 \mathrm{~mm} / 37 \mathrm{~mm}\end{array}$ & Number of beams & $128 / 128$ \\
\hline $\begin{array}{l}\text { Dynamic receive } \\
\text { focusing }\end{array}$ & Yes / yes & F-number on receive & $1.1 / 1,1$ \\
\hline Elevation focus & $50-60 \mathrm{~mm} / 50-70 \mathrm{~mm}$ & scan depth & $60 \mathrm{~mm} / 103,5 \mathrm{~mm}$ \\
\hline Transmit apodization & Hamming (full aperture) /None & Opening angle & $-20^{\circ}$ to $20^{\circ} /-30^{\circ}$ to $30^{\circ}$ \\
\hline
\end{tabular}

Lagrangian-Eulerian mesh motion [51] defined on all interior nodes ensured that the mesh quality was preserved, while avoiding smoothing and remeshing. For further details, we refer to [45]. The Navier-Stokes equations were numerically solved with a finite volume method, and took $14 \mathrm{~h}$ and 50 min on a remote eight-core $64-\mathrm{b} 3.4-\mathrm{GHz}$ processor to acquire three full cardiac cycles. Blood was modeled as a Newtonian fluid, with a dynamic viscosity of $5.5 \mathrm{mPa} \cdot \mathrm{s}$ according to the neonatal range reported in [52] and a density of 1060 $\mathrm{kg} / \mathrm{m}^{3}$. The 3-D intraventricular blood flow was obtained over an entire cardiac cycle (120 beats/min) at a frame rate of 200 $\mathrm{Hz}$, and is shown during the end of the rapid filling phase in Fig. 2(c).

\section{B. Ultrasonic Simulation}

The apical four-chamber view was mimicked by orienting the apex of the left ventricular model toward the probe, a 96-element 1-D phased-array transducer for pediatric cardiology, which is $14 \mathrm{~mm} \times 10 \mathrm{~mm}$ in the lateral and elevational directions, respectively, with a pitch of $150 \mu \mathrm{m}$ and kerf of $30 \mu \mathrm{m}$. To ensure that all point scatterers were situated in the simulation's far field, the elevational width of each transducer element was divided into four mathematical elements. The probe had a center frequency of $6 \mathrm{MHz}$ with a relative bandwidth of $75 \%$ and a fixed elevational focus at $50-\mathrm{mm}$ depth.

The full aperture was used to transmit a single diverging wave at a Pulse Repetition Frequency (PRF) of $9 \mathrm{kHz}$. No elevational nor lateral tilt was applied, while the virtual source was placed $13.3 \mathrm{~mm}$ behind the transducer's surface, corresponding to a $60^{\circ}$ opening angle. Two pulse periods were transmitted as a compromise between penetration depth and axial resolution, and a Hamming apodization scheme was used on transmit. The pulse echo responses of 50 consecutive transmissions (a packet) were used to acquire one 2-D velocity map (see Section II-C). Within a packet, the scatterers were propagated according to the spatially interpolated CFD velocities [37], while the scatterer ensemble was refreshed in between packets to avoid clustering or dilution. Approximately ten scatterers per resolution cell ensured a Gaussian-distributed RF signal [53].

Channel data were acquired with Field II [13], [43] for the full cardiac cycle at a fast time sampling rate of $100 \mathrm{MHz}$, subsequently demodulated at $5.63 \mathrm{MHz}$, and eventually resampled to $48 \mathrm{MHz}$ before beamforming. All Field II simulations were performed in parallel on a cluster-computing infrastructure consisting of dual-socket octacore Intel Xeon Sandy Bridge (E5-2670) 2.6-GHz processors, and took $20 \mathrm{~h}$ for one packet.

Only the receive beams within a sector defined by an opening angle of $40^{\circ}$ were used for further processing due to reduced intensity at the edges of the field of view. A sum-anddelay beamformer reconstructed 128 equally spaced receive beams. Dynamic receive focusing $(\mathrm{F}$-number $=1.1)$ assured an optimal image quality, while a Hamming apodization scheme on receive reduced the lateral sidelobes. Finally, adding Gaussian noise at an SNR of $20 \mathrm{~dB}$ and applying a fourth-order polynomial regression filter with a $-3-\mathrm{dB}$ cutoff at $4.8 \mathrm{~cm} / \mathrm{s}$ enhanced the realism of the results. Note that as this clutter filter directly works on the slow-time domain of the acquired In-phase and Quadrature (IQ) data (i.e., in the packet 
range), the filter cutoff refers to the $-3 \mathrm{~dB}$ attenuation of IQ signals with a phase shift corresponding to an axial velocity of below $4.8 \mathrm{~cm} / \mathrm{s}$. An overview of all imaging settings can be found in Table I.

\section{Velocity Estimation}

ST was performed by finding the best match of a discrete set of 98 lateral $\times 100$ axial kernels within a surrounding search region defined in a subsequent acquisition using the sum of absolute difference (SAD) algorithm. The optimal kernel size was found to be $0.25 \mathrm{~mm} \times 0.25 \mathrm{~mm}$ after performing a parametric study, varying the kernel size from 0.1 to $1.5 \mathrm{~mm}$. Subpixel accuracy was obtained by parabolic interpolation on the SAD matrix. Based on the ground-truth CFD velocities, the maximal trackable velocities were defined as $0.8 \mathrm{~m} / \mathrm{s}$ laterally and $1.2 \mathrm{~m} / \mathrm{s}$ axially, resulting in a search region of $0.43 \mathrm{~mm} \times 0.52 \mathrm{~mm}$. The minimal trackable velocities in ST are defined by the lateral and axial sampling of the IQ data, which we increased by spline interpolation with a factor of 15 in the lateral direction, resulting in $0.15 \mathrm{~m} / \mathrm{s}$ in both directions. Note that this is still a quite high resolution velocity, but choosing lower values would result in a huge increase in computational time. Furthermore, there is also a limit to boosting the spatial sampling of the data as it does not necessarily mean that extra information is gained. We investigated both increased interpolation of the IQ data as well as a higher number of receive beams: our final setup was a tradeoff between the computational time and the limited info added when increasing spatial sampling. Finally, this resulted in a velocity map with a lateral sampling distance between 0.11 (proximal to the probe) and $0.43 \mathrm{~mm}$ (distal to the probe) and an axial sampling distance of $0.45 \mathrm{~mm}$, where we removed estimates tracked outside the blood pool. To reduce the effect of spurious detection errors, the median averaged velocity was computed over an ensemble of 50 acquisitions, resulting in a frame rate of $180 \mathrm{~Hz}(=\mathrm{PRF} / 50)$ for the reconstructed 2 -D velocity images. Note that the timespan to generate one 2 -D velocity image $(1 / 180 \mathrm{~Hz}=5.6 \mathrm{~ms})$ is very close to the time step used in the CFD simulations $(5 \mathrm{~ms})$. Hence, the displacement field of the scatterers will hardly change during one observation window though one or two updates in the displacement field might occur, depending on the exact timing of scanning. However, the random distribution of scatterers is changing over time due to the complex 3-D displacement field, affecting the mutual positioning of these points over time. As such, the speckle pattern might decorrelate during one acquired packet due to out-of-plane flow or strong spatial gradients.

Spatial averaging and temporal averaging were applied to smoothen these 2-D ST velocity maps (at $180 \mathrm{~Hz}$ ): a spatial median filter over $5 \times 5$ kernels and a backward weighted averaging temporal filter

$v_{t}=\frac{1}{15} v_{t-4 \Delta t}+\frac{2}{15} v_{t-3 \Delta t}+\frac{3}{15} v_{t-2 \Delta t}+\frac{4}{15} v_{t-\Delta t}+\frac{5}{15} v_{t}$.

The performance of ST was quantified by comparing the ground truth with the estimated velocities. Relevant perfor-



Fig. 3. (A) In vitro phantom consisted of a smaller chamber representing the left atrium and a larger chamber representing the left ventricle. (B) B-mode image indicating the interior dimensions of the heart-mimicking chambers (left ventricle and left atrium).

mance estimators, such as the deviation in velocity magnitude $\left(\Delta V_{\text {mag }}\right)$ and direction $(\Delta \varangle)$, were calculated. These estimators have, however, a highly skewed distribution, requiring robust measures of central tendency and variability. Hence, median values as central tendency and median absolute deviation (MAD) as variability will be reported. The MAD of the deviation in estimated velocity magnitude (3) and direction (4) will be reported and calculated as follows:

$$
\begin{aligned}
\operatorname{MAD}(V \operatorname{mag}) & =\operatorname{median}\left(\left|\Delta V_{\text {mag }}-\operatorname{median}\left(\Delta V_{\text {mag }}\right)\right|\right) \\
\operatorname{MAD}(\varangle) & =\operatorname{median}(|\Delta \varangle-\operatorname{median}(\Delta \varangle)|) .
\end{aligned}
$$

\section{In Vitro Experiments}

To demonstrate the realism of the simulated 2-D velocity maps, we also performed in vitro experiments mimicking a diastolic inflow jet. In particular, our aim was to verify whether the artifacts observed in the simulations were independent of the simulation methodology and would also arise in real life. This jet flow phantom [Fig. 3(a)] made from Plexiglas consisted of a small chamber, representing the left atrium, and a large chamber, representing the left ventricle, thereby mimicking the diastolic inflow jet in the adult case. Both chambers were separated by a membrane with a hole of 8-mm diameter, resembling the mitral valve in open position [interior dimensions indicated in Fig. 3(b)]. Pressurization of the fluid on the atrial side resulted in a jet flow with velocities in the range of $0.8-1 \mathrm{~m} / \mathrm{s}$. A low-viscous bloodmimicking fluid was used to resemble the scattering properties of blood and consisted of tap water, with $9 \mathrm{wt} \%$ dissolvent and $1.82 \mathrm{wt} \%$ nylon particles.

A high frame rate diverging wave acquisition was implemented on the Verasonics Vantage platform (Verasonics, Inc., Kirkland, WA, USA). The P4-2V adult cardiac phased-array probe (Verasonics, Inc., Kirkland, WA, USA) was used with 64 elements and a center frequency of $2.98 \mathrm{MHz}$. Further details on the probe and the scanning setup can be found in Table I. Channel data were acquired at a sampling frequency of $11.92 \mathrm{MHz}$, beamformed, and IQ demodulated. The increased imaging depth lowered the PRF to $6 \mathrm{kHz}$. Different ST and clutter filter settings were required for the in vitro acquisitions. A third-order polynomial regression filter ensured a similar $-3-\mathrm{dB}$ cutoff of $0.049 \mathrm{~m} / \mathrm{s}$. The lower spatial resolution in the speckle images required to enlarge the kernel size to $1.0 \mathrm{~mm} \times 1.0 \mathrm{~mm}$, with search regions of $1.3 \mathrm{~mm} \times 1.3 \mathrm{~mm}$ resulting in trackable velocities 


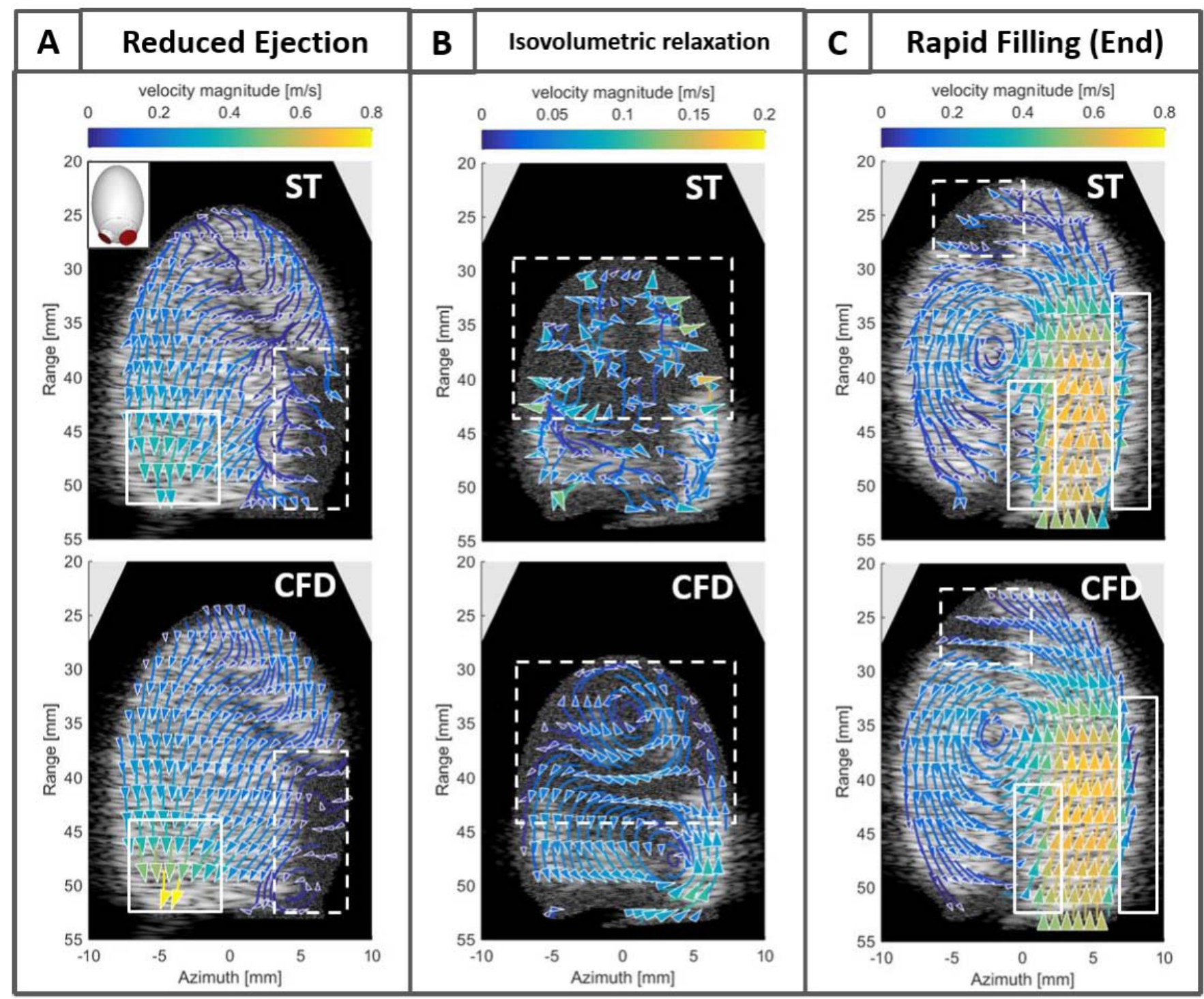

Fig. 4. Examples of the CFD and ST vector flow field at three instances in time. (A) Reduced ejection phase. (B) Isovolumetric relaxation phase. (C) Rapid filling phase. Notable artifacts are indicated by the white boxes in both the CFD and ST results.

of $1 \mathrm{~m} / \mathrm{s} \times 1 \mathrm{~m} / \mathrm{s}$. The resulting velocity map was sampled at 1 $\mathrm{mm}$ in both the lateral and axial directions. Spatial smoothing and temporal smoothing were performed in a similar fashion as the simulations.

\section{RESUlts}

\section{A. Simulation Results}

Fig. 4 shows both ST flow fields (top) with their respective CFD ground-truth counterparts (bottom) at three cardiac phases, as indicated by the arrow boxes in Fig. 2(b), i.e., the reduced ejection phase [Fig. 4(a)], the isovolumetric relaxation phase [Fig. 4(b)], and the end of the rapid filling phase [Fig. 4(c)]. The in-plane vectorial results are color encoded with their velocity magnitude, whereas the background shows the speckle image on which ST was performed. The most noteworthy observed artifacts are indicated by the white boxes, and will be further discussed in the following.

1) Panel A: The reduced ejection phase portrays two distinct artifacts. The first one is indicated by the dashed boxes in Fig. 4(a) and corresponds to zones of low flow, or as can be seen from the speckle images, to zones where the blood flow signal has been removed by the clutter filter. A large average deviation in a flow direction of $74.7^{\circ}$ (MAD 36.3 $)$ was noted in this zone, compared with the overall deviations in a flow angle of $15.7^{\circ}$ (MAD $12.4^{\circ}$ ). The magnitude of the flow in this zone was on average overestimated by $4.4 \mathrm{~cm} / \mathrm{s}$ (MAD $2.7 \mathrm{~cm} / \mathrm{s}$ ), compared with an underestimation of $2.7 \mathrm{~cm} / \mathrm{s}($ MAD $2.2 \mathrm{~cm} / \mathrm{s}$ ) or $27 \%$ (normalized to the mean CFD velocity magnitude) over the full field of view. The second artifact, persisting throughout systole, is displayed in the solid boxes in Fig. 4(a) or in greater detail for ST and CFD in Fig. 5(a) and (b), respectively. The high blood flow velocities up to $1.2 \mathrm{~m} / \mathrm{s}$ near the aortic outlet are severely underestimated by ST. Despite these large magnitude underestimations, the direction of the flow was accurately tracked with a limited deviation in flow angle of $6^{\circ}\left(\mathrm{MAD} 2.9^{\circ}\right)$ in that zone. 




Fig. 5. (A) Estimated and (B) ground-truth flow pattern during the reduced ejection phase. (C) Velocities normal to the surface, defined by the white dashed line in $(\mathrm{A})$ and $(\mathrm{B})$, and the elevation direction.



Fig. 6. (A) Estimated and (B) ground-truth flow pattern during the end of the rapid filling phase with the jet flow artifact indicated by the white solid boxes. Velocities along the white dashed line in (A) and (B) are given for (C) axial component and (D) lateral component.

2) Panel B: During the isovolumetric relaxation phase, the blood flow signal is filtered out almost completely [Fig. 4(b)]. The low velocity vortical flow patterns observed in the CFD flow pattern are not distinguishable anymore in the ST estimates. Overall, a large deviation in directionality of $53^{\circ}$ (MAD $27.5^{\circ}$ ) is observed. The magnitude of the flow pattern deviates only slightly $(2.7 \mathrm{~cm} / \mathrm{s}$, MAD $1.5 \mathrm{~cm} / \mathrm{s})$; however, normal- ized to the mean CFD velocity magnitude at that time instance, it does portray significant magnitude deviations of $80 \%$.

3) Panel $C$ : The third phase observed in greater detail is the rapid filling phase [Fig. 4(c)]. The dashed boxes again indicate zones where the blood signal was lost. An additional artifact is noted at the edges of the mitral inflow jet (solid boxes), displayed in greater 


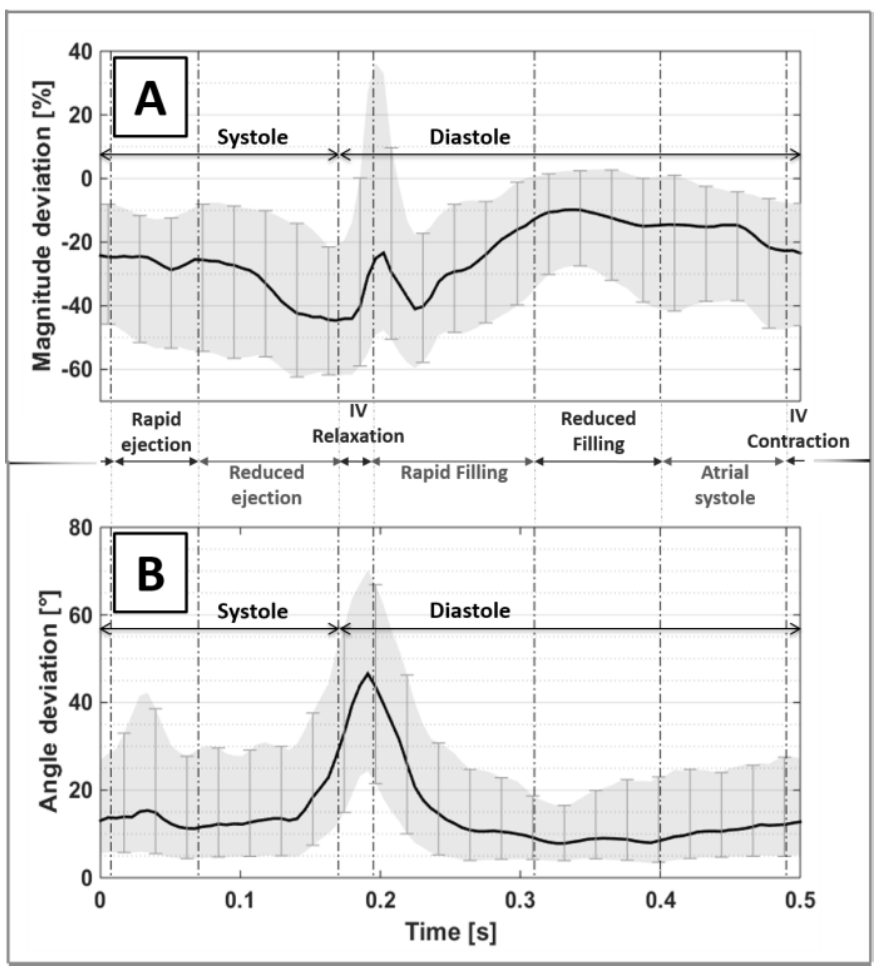

Fig. 7. (A) Relative deviation in estimated velocity magnitude. (B) Absolute deviation in flow direction. The cardiac phases are indicated by the vertical dashed-dotted lines. The solid black lines represent the median values over the full field of view, while the gray zones represent the interquartile distance.

detail in Fig. 6(a) and (b). The velocity profiles across the white dashed line in these panels are portrayed in Fig. 6(c) and (d), showing, respectively, the axial and lateral velocities across the jet. Looking at the axial component [Fig. 6(c)], one can note the excellent match of the estimates with respect to the CFD velocities. Within the extent of the jet, the axial velocities are underestimated on average by $4.8 \%$ (MAD 6.8\%). The lateral components on the contrary [Fig. 6(d)] show large deviations. In particular, one can observe that in a zone of 1-3 mm adjacent to the main jet (left side), the ST algorithm erroneously estimates the lateral velocity component to be negative (opposed to positive in the CFD ground truth), resulting in the full 2-D velocity vector to be drawn away from the center of the jet. The opposite occurs within the jet zone, bounded by the dashed-dotted line in Fig. 6(c) and (d): at the edges of the jet core, the lateral velocity component is overestimated on the left side while underestimated on the right side, resulting in velocity vectors to be drawn toward the center of the jet.

A full analysis over the cardiac cycle is depicted in Fig. 7, demonstrating the relative deviation in estimated velocity magnitudes [Fig. 7(a)] and the deviations for the in-plane vectorial angles [Fig. 7(b)]. The solid black line represents the median value for the complete field of view, while the error bars indicate the first and third quartiles. To enhance interpretation of these results, the respective phases of the cardiac cycle are indicated with dashed lines. The blood flow magnitude
[Fig. 7(a)] is always underestimated, while the deviations in estimated blood flow direction [Fig. 7(b)] are below $15^{\circ}$ for most of the cardiac cycle. During the systolic phase, the estimated magnitude deteriorates progressively, peaking at a deviation of $-44.6 \%$ (MAD $19.7 \%$ ) at the end of the reduced ejection phase. The isovolumetric relaxation phase leads to a decrease in median error, but is, however, also accompanied by a higher spread in deviations (MAD 44.4\%), notable by the high interquartile distance in Fig. 7(a). High angular deviations are also observed during this phase, which is in correspondence with the reported values for Fig. 4(b). The estimated magnitude is subsequently found to improve up to the start of the reduced filling phase (9.8\%, MAD 13.7\%), followed by a slight decay up to the start of the systolic phase. A similar evolution is observed for the deviations in flow direction, with a minimal average deviation of $7.9^{\circ}$ $\left(\mathrm{MAD} 5^{\circ}\right)$ at the start of the reduced filling phase, and slightly deteriorating afterward.

To further provide a statistical quantitative analysis of the ST performance, a linear regression analysis is presented for the systolic (reduced ejection) and diastolic phase (rapid filling) in Fig. 8. Scatter plots show the relationship between the estimated axial and lateral velocity component versus the CFD ground truth in, respectively, the top and bottom panels. The pink cross symbols indicate the velocities below the clutter filter cutoff, and the black circles refer to velocities above this cutoff. The regression analysis on the velocities above the clutter filter cutoff is represented by the red dashed line. Outliers more than three standard deviations from the mean were removed from the data set. ST is obviously a better axial than lateral flow estimator, with less spurious errors present for the top than bottom panels. Indeed, the $R^{2}$ value for the axial estimates is, respectively, 0.94 and 0.96 for systole and diastole, versus 0.33 and 0.53 for the lateral estimates at these two time instances.

\section{B. In Vitro Results}

To verify whether the artifact reported for the diastolic inflow jet in Figs. 4(c) and 5 was realistic and independent of any simulation settings, we analyzed the vector flow maps of the in vitro measurement in Fig. 9(a) and directly compared the result with the simulation data. As can be noted from these results, a large lateral velocity component is estimated next to the jet, indicated by the white solid boxes in Fig. 9(a). Similar to Fig. 6 for the simulations, Fig. 9(b) and (c) indicates, respectively, the axial and lateral estimated velocities along the white dotted line in Fig. 9(a). Furthermore, the simulation results reported along the line in Fig. 6(c) and (d) are plotted on the top (dashed lines), but are scaled for this purpose to the extent of the in vitro jet. Qualitatively, we observe the same tracking artifact as in the simulations. Indeed, ST results erroneously show the blood flow within the extent of the jet (indicated by the vertical dashed lines) to be drawn toward the jet center [arrows in Fig. 9(c)], while outside the jet, the blood flow is drawn away from the jet [arrows in Fig. 9(c)]. Quantitatively, however, the jet distortion is more severe for the in vitro flow results. The lateral velocity deviations within the extent of the jet vary from -0.15 to $0.49 \mathrm{~m} / \mathrm{s}$ for the in vitro 

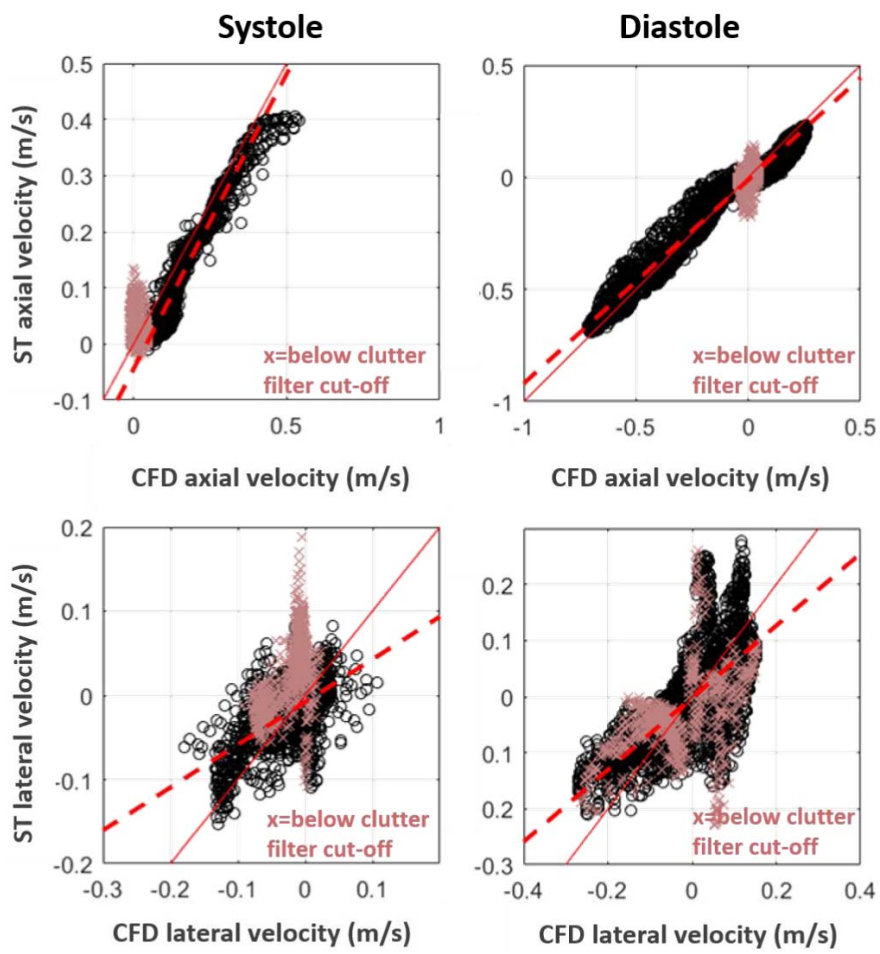

Fig. 8. Linear regression analysis is presented for the systolic (reduced ejection) and diastolic phases (rapid filling). Scatter plots show the relationship between the estimated axial and lateral velocity component versus the CFD ground truth in, respectively, the top and bottom panels. The pink cross symbols indicate the velocities below the clutter filter cutoff, and the black circles refer to velocities above this cutoff. The regression analysis on the velocities above the clutter filter cutoff is represented by the red dashed line. Outliers more than three standard deviations from the mean were removed from the data set.

acquisitions, while the simulations only portray deviations of -0.21 to $0.2 \mathrm{~m} / \mathrm{s}$ in that zone. Outside the jet, lateral velocity deviations up to $\pm 1 \mathrm{~m} / \mathrm{s}$ are noted for the in vitro results, while only deviations of $\pm 0.18 \mathrm{~m} / \mathrm{s}$ are noted for the simulations.

\section{DISCUSSION}

This paper provided an in-depth quantitative analysis of high frame rate vector flow imaging in the neonatal cardiac setting. Computational modeling findings were supported by additional in vitro experiments, which confirmed the erroneous velocity estimation at the edge of the diastolic inflow jet observed in the simulations. The results showed that during most of the cardiac cycle, the flow pattern could be clearly recognized and deviations in flow magnitude and direction were acceptable. However, deteriorated velocity measurements were identified during particular instances and in specific zones of the ventricular model, requiring further discussion of the results.

\section{A. Artifact at the Edges of the Diastolic Inflow Jet}

At the boundaries of the jet zone, velocities were corrupted due to an erroneous estimation of the lateral velocities. At the edges of the jet, a transitional zone arose where blood flow was seemingly drawn away from the jet at its outer skirts while flow was drawn inward when closer to the jet core.



Fig. 9. (A) ST results of the in vitro flow show distinctive artifacts at the edges of the jet (white solid boxes). The velocity components along the white dashed line in (A) (black solid lines) are compared with the simulation results (black dashed lines) of (B) axial velocity and (C) lateral velocity.

A very similar distortion at the edges of the jet appeared for the in vitro results, which validated the findings of our simulation environment. Note that quantitative differences in velocity magnitude between the simulations and in vitro results were present, and were likely caused by a variety of confounding factors: 1) a different velocity profile, higher in magnitude and more parabolic in the case of the experiment; 2) a different probe and scanning settings (Table I); and 3) different beamforming techniques (Verasonics versus in-house sum-and-delay beamformer). Nevertheless, a qualitative match was convincingly retrieved for the lateral velocity distortion [Fig. 9(c)].

This phenomenon was previously noted by Fadnes et al. [33] in their model of neonatal ventricular shunt flow, though the image acquisition relied on plane waves. Their parabolic shunt velocity profile caused high spatial velocity gradients over most the shunt, similarly resulting in inward drawn blood flow at the edges of the jet. Strong sidelobes produced by plane wave transmissions were considered to cause a bias in the lateral velocity estimates when slowly moving scatterers were adjacent to fast moving scatterers. As Tong et al. [54] indicated, diverging waves have significantly higher beamwidth and sidelobe to main lobe ratio than focused beams, both aspects disfavoring diverging waves for picking up spatial velocity gradients in the lateral direction.

As can be seen from Fig. 6, these distortions correspond to zones of high spatial gradients of the velocity magnitude in the lateral direction, as a rapid transition of high velocity magnitude in the jet core (red) to the low velocity magnitudes in the jet surroundings (blue) is present. It appears that when a kernel is positioned close to a peak in the velocity gradient, the match is dominated by the side of the kernel covering the lower part of the gradient, where shearing is lowest. The question is, 




Fig. 10. (A) Axial and (B) lateral velocity components across a horizontal line, as depicted in Fig. 6 (white dashed line), for various imaging schemes: diverging waves in a black dashed line, plane waves in a gray solid line, and focused beams in a gray dashed line. The black solid line represents the ground-truth velocity components.

however, to what extent the flow physics are responsible for this artifact and whether the ultrasound imaging setup also plays a role. For this purpose, additional simulations were performed to investigate whether the transmission of diverging waves contributed to this phenomenon and whether different scanning schemes could improve the result. An imaging setup relying on plane waves and focused beams (focal point at $50-\mathrm{mm}$ depth) was studied, with the velocity estimation procedure identical as in the case of diverging waves. Note that the focused beam acquisition consisted of an equal number of transmit beams as the number of receive beams used for plane and diverging wave emissions. The imaged scatterer propagation for focused imaging was taken identical to the high frame rate imaging simulations of diverging and plane waves. Fig. 10 indicates the lateral and axial velocities along a horizontal line at $50-\mathrm{mm}$ depth for the transmission of diverging waves (bold dashed lines), plane waves (gray solid lines), or focused beams (gray dashed line), compared with the CFD ground truth (bold solid line). We found that the imaging setup hardly affected the estimation of the main jet velocities (axial components), but the lateral velocities showed larger deviations between the different scanning schemes. Despite the quantitative differences, all three imaging sequences showed the same pattern of distortion, with inward drawn components toward the center of the jet and outward drawn components at the outer edge of the jet. Focused beams showed the smallest distortions, almost negligible in the center of the jet $(<0.04 \mathrm{~m} / \mathrm{s}$ between 2 and $4.5 \mathrm{~mm}$ azimuth). The distortion for plane waves appeared to be similar, yet slightly larger in magnitude compared with the diverging wave distortion, confirming the work in [33].

To further investigate the contribution of the ultrasound imaging physics, we performed additional simulations with the ventricle $90^{\circ}$ rotated, i.e., the parasternal long-axis view compared with the previous apical four-chamber view. This means that we will be imaging the diastolic inflow jet along the lateral direction [Fig. 11(a)], though with an identical imaging setup. As there is an inherent mismatch in ultrasound imaging between the lateral and axial imaging resolutions, one would expect different results when the flow field is

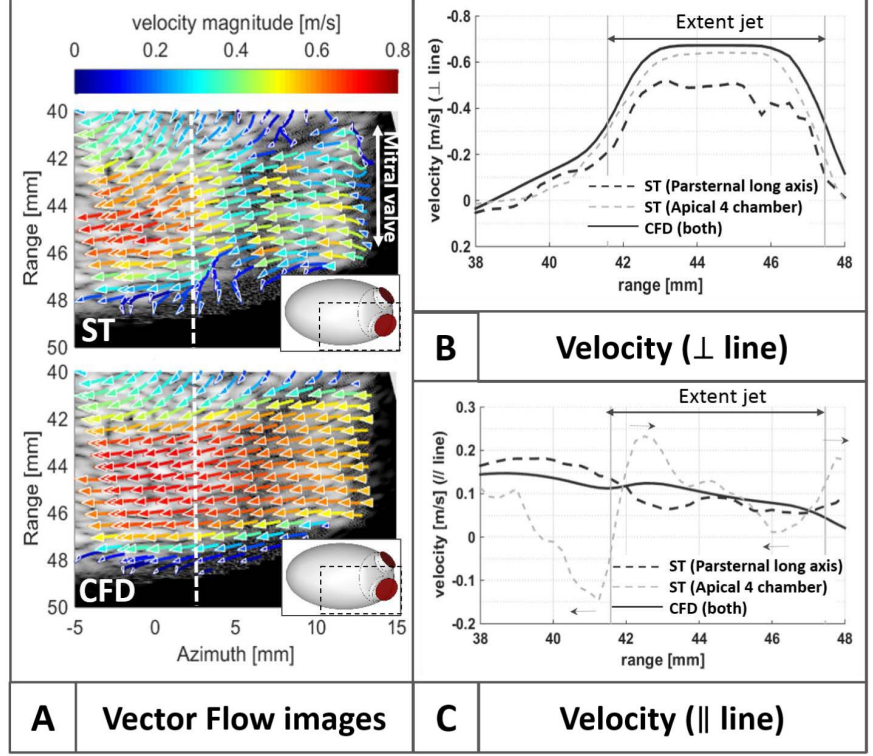

Fig. 11. (A) Vector flow results at the end of the rapid filling phase, yet now the phantom is oriented in a parasternal long-axis view. The in-plane velocities across the white dashed line in (A) are given for (B) perpendicular and (C) parallel components.

rotated. In Fig. 11, the area across the jet now corresponds to a vertical white dashed line in Fig. 11(a). The estimated velocities perpendicular $(\perp)$ and parallel $(\|)$ to that line are, respectively, plotted in Fig. 11(b) and (c) with a black dashed line, while the solid black lines represent the ground-truth CFD velocities. We directly compared these results with the estimates from the original apical four-chamber orientation of the phantom (gray dotted line), which are presented in Fig. 6. Proper comparison, however, required that, due to the rotation, the axial velocity estimates in Fig. 6 were compared with the lateral velocities of the parasternal view in Fig. 11(b), and vice versa for Fig. 11(c). Rotating the phantom $90^{\circ}$ clearly caused a larger underestimation of the main component of the jet velocities [Fig. 11(b)]. However, the parasternal view alleviated the previously described artifact at the boundaries of the jet 
[black dashed versus gray dotted line in Fig. 11(c)]. This was indeed to be expected as lateral velocity estimates are consistently worse than axial estimates, considering the significantly better axial resolution, hampering accurate assessment of strong flow gradients in the lateral direction. Due to this mismatch in axial and lateral resolutions, the cardiac view affects the accuracy of the portrayed jet, keeping in mind that a jet with its main flow core along the lateral direction is distorted less. However, this view also resulted in a larger volume flow underestimation due to a worse estimate of the main jet velocity component. Indeed, the volume flow underestimation (per unit of elevation width) is $8.5 \%$ in the case of the apical four-chamber view, while it is significantly higher in the parasternal long-axis view (29.6\%).

\section{B. Artifact in Low Flow Zones}

Large deviations in the blood velocity estimates were present in the low flow zones due to the applied signal processing as the clutter filter strongly attenuated the IQ data corresponding to axial velocities below $0.048 \mathrm{~m} / \mathrm{s}$. Furthermore, the minimally trackable velocity in both the lateral and axial directions was quite high $(0.15 \mathrm{~m} / \mathrm{s})$, as this resulted from a tradeoff between upsampling of the IQ data and keeping reasonable computational times. This meant that a quite large region of the ventricle suffered from deteriorated estimates, in particular during the isovolumetric relaxation (Fig. 7), leading to unrecognizable flow patterns [Fig. 4(b)]. Indeed, this time instance corresponds to the ventricular outflow or inflow velocities dropping to (almost) zero [Fig. 2(b)], though this is also the case during the reduced filling phase and the isovolumetric contraction. However, the diastolic vortex created by the mitral inflow [Fig. 4(c)] will generate sufficient intraventricular flow during these latter two phases (at least for the simulated conditions), resulting in flow above the clutter filter cutoff ( $>0.048 \mathrm{~m} / \mathrm{s})$.

Lowering the cutoff value of the clutter filter might not be a good idea, as the surrounding cardiac tissues will not likely be filtered out [55], but adaptive clutter filtering [56], [57] might offer a solution, providing low cutoffs at instances of low tissue velocities and high cutoffs at instances of high tissue velocities. Furthermore, one should bear in mind that such low flow velocities are difficult to track when using a relatively high PRF of $9 \mathrm{kHz}$, resulting in a minimal detectable velocity of $0.15 \mathrm{~m} / \mathrm{s}$ after laterally interpolating the IQ data with a factor of 15 . Artificially lowering the PRF by skipping frames during the tracking procedure might boost the estimation accuracy in these cases.

\section{Artifact in Systolic Outflow Region}

During the entire systolic phase [Fig. 4(a)], the measured velocities with ST significantly differed from the ground truth, in particular near the aortic outlet (Fig. 5). The normal velocity component along the white dashed line in Fig. 5(a) and (b) is shown in Fig. 5(c) and portrayed underestimations from $14.5 \%$ up to $64.7 \%$. This would lead to a severe error in measured outflow of $-44 \%$ compared with the ground truth. The reason for this severe underestimation is to be found in the decorrelation of the speckle pattern due to the complex flow physics and plays at two levels. As the systolic ejection pushes the blood toward the aortic outlet, it creates: 1) high velocity gradients and 2) considerable out-of-plane flow components up to $0.57 \mathrm{~m} / \mathrm{s}$ [Fig. 5(c)] due to the asymmetry in the model. Both lead to speckle decorrelation [18], impeding accurate velocity measurements. A severe underestimation of high velocities was also noted by Gao et al. [22] for 3-D ST in their model of the adult left ventricle, although during different cardiac phases. 3-D ST is, however, not hampered by out-of-plane flow components, and might provide better estimates and volume flow estimations.

\section{Limitations and Future Work}

The main benefit of the presented multiphysics simulations was to dispose of a fully known ground-truth flow field (CFD) when validating these cardiac flow imaging strategies and signal processing, typically lacking during in vitro or in vivo validation. However, one can expect a large variety of neonatal ventricular flow fields depending on birth weight and the presence of potential defects, and it was not our aim to cover all these hemodynamic conditions and their effect on the resulting images in this paper. Furthermore, the CFD simulations relied on a generic model of neonatal left ventricle, mathematically described by a truncated prolate spheroid with valves modeled either fully open or closed. To create a more physiological model, a fluid-structure interaction simulation approach [58], [59] based on 3-D segmentation of a ventricular cavity and detection of its wall motion based on patientspecific images could be considered. However, the advantage of our mathematical formulated model lies in its ease of use and versatility, allowing us to vary important cardiac parameters like the EF or the EDV. The choices of these parameters are constricted only by geometrical design, i.e., the truncation radius $(r)$ needs to be larger than half the longaxis length (c) and sufficiently large to allow both the inlet and outlet to be placed on the top.

In addition, incorporating surrounding tissues, i.e., the surrounding cardiac muscle, the valves, or chordae tendineae, would further enhance the realism of the model [38]. The RF signals originating from these tissues are several tens of decibels stronger than the RF signal of blood, and might cause strong sidelobes and specular reflections protruding into the region of blood flow, hampering the blood velocity estimation adjacent to the cardiac tissues. For now, we investigated the effect of the clutter filter on the blood velocity estimation, but neglected the effect of the cardiac wall movement.

The increase in computational power has also made it possible to optimize tracking results by employing NavierStokes-based regularization algorithms and dynamic kernel sizes based on histogram properties, as indicated in [60]. Other Navier-Stokes-based approaches attempt to reconstruct the 2-D [61]-[64] or 3-D [65] flow pattern from the 1-D CFI images. Such regularization algorithms might benefit from using the normalized cross correlation instead of the SAD 
algorithm as it provides a quality measure of the obtained estimates, indicating zones with inferior velocity data. Despite being fast, assumptions on the flow pattern are necessary (laminar, in-plane flow, appropriate boundary conditions, etc.), restricting the general applicability of such techniques. Furthermore, tracking performance could be improved by considering kernel shapes different from the square ones we used, rather mimicking the shape of the point spread function, as well as choosing a dynamic kernel size depending on the cardiac phase. Increased computational power also opens up the possibility of 3-D ST [22] for blood flow estimation. The out-of-plane flow restriction in 2-D, which had a significant impact on our results obtained during the systolic phase, might as such be resolved.

The identification of artifacts, related to the imaging scheme and/or the underlying velocity flow field, might be the onset for automatic detection and removal of such artifacts. Imaging schemes might also be optimized in such a way that artifacts are minimized. Either way, computational multiphysics approaches will continue to play an important role for the initial research and validation as it starts from a known velocity field.

\section{CONCLUSION}

Our computational multiphysics modeling approach enabled us to quantitatively analyze the performance of 2-D ST on intraventricular flow in neonates when transmitting single diverging waves at an ultrafast frame rate of $9 \mathrm{kHz}$. Specific flow conditions proved, however, to be detrimental: 1) very low flows; 2) high spatial flow gradients; and 3) large outof-plane flow resulted in large biases. At the edges of the jet, a transitional zone arose where blood flow was seemingly drawn away from the jet at its outer skirts, while flow was drawn inward when closer to the jet core, an artifact also obtained in an in vitro setting. The flexibility of our simulation environment allowed us to pinpoint to what extent these artifacts arose due to the signal processing, the imaging setup, or the complex 3-D flow physics. Despite these artifacts, a good estimation of the 2-D intraventricular flow pattern was still obtained at a frame rate of $180 \mathrm{~Hz}$.

\section{REFERENCES}

[1] P. P. Sengupta et al., "Emerging trends in CV flow visualization," JACC, Cardiovascular Imag., vol. 5, no. 3, pp. 305-316, 2012.

[2] D. J. Fillipps and R. L. Bucciarelli, "Cardiac evaluation of the newborn," Pediatric Clin. North Amer., vol. 62, no. 2, pp. 471-489, 2015.

[3] W. W. Lai et al., "Guidelines and standards for performance of a pediatric echocardiogram: A report from the task force of the pediatric council of the American society of echocardiography," J. Amer. Soc. Echocardiogr., vol. 19, no. 12, pp. 1413-1430, 2006.

[4] P.-L. Bernier, A. Stefanescu, G. Samoukovic, and C. I. Tchervenkov, "The challenge of congenital heart disease worldwide: Epidemiologic and demographic facts," Seminars Thoracic Cardiovascular Surgery, Pediatric Cardiac Surgery Annu., vol. 13, no. 1, pp. 26-34, 2010.

[5] Eurocat Prevalance Data Tables, Eurocat, Via E. Fermi 2740, I-21027 ISPRA (VA), Italy, 2012.

[6] O. J. Benavidez, K. Gauvreau, K. J. Jenkins, and T. Geva, "Diagnostic errors in pediatric echocardiography: Development of taxonomy and identification of risk factors," Circulation, vol. 117, no. 23, pp. 2995-3001, Jun. 2008.
[7] M. S. Chew and J. Poelaert, "Accuracy and repeatability of pediatric cardiac output measurement using Doppler: 20-year review of the literature," Intensive Care Med., vol. 29, no. 11, pp. 1889-1894, Nov. 2003.

[8] G. Pedrizzetti and F. Domenichini, "Nature optimizes the swirling flow in the human left ventricle," Phys. Rev. Lett., vol. 95, no. 10, p. 108101, Sep. 2005.

[9] H. Oertel, K. Spiegel, and S. Donisi, "Modelling the human cardiac fluid mechanics," Dept. Flow Mech. (Institüt fur Strömungsmechanik), Univ. Karlsruhe, Karlsruhe, Germany, Tech. Rep. 978-3-86644-087-6, 2006.

[10] F. Domenichini, G. Querzoli, A. Cenedese, and G. Pedrizzetti, "Combined experimental and numerical analysis of the flow structure into the left ventricle," J. Biomech., vol. 40, no. 9, pp. 1988-1994, 2007.

[11] M. D. Fox, "Multiple crossed-beam ultrasound Doppler velocimetry," IEEE Trans. Sonics Ultrason., vol. SU-25, no. 5, pp. 281-286, Sep. 1978.

[12] B. Dunmire, K. W. Beach, K.-H. Labs, M. Plett, and D. E. Strandness, "Cross-beam vector Doppler ultrasound for angle-independent velocity measurements," Ultrasound Med. Biol., vol. 26, no. 8, pp. 1213-1235, 2000.

[13] J. A. Jensen and P. Munk, "A new method for estimation of velocity vectors," IEEE Trans. Ultrason., Ferroelectr., Freq. Control, vol. 45, no. 3, pp. 837-851, May 1998.

[14] I. K. Ekroll, A. Swillens, P. Segers, T. Dahl, H. Torp, and L. Lovstakken, "Simultaneous quantification of flow and tissue velocities based on multi-angle plane wave imaging," IEEE Trans. Ultrason., Ferroelectr., Freq. Control, vol. 60, no. 4, pp. 727-738, Apr. 2013.

[15] J. Udesen, M. B. Nielsen, K. R. Nielsen, and J. A. Jensen, "Examples of in vivo blood vector velocity estimation," Ultrasound Med. Biol., vol. 33, no. 4, pp. 541-548, Apr. 2007.

[16] P. M. Hansen et al., "Volume flow in arteriovenous fistulas using vector velocity ultrasound," Ultrasound Med. Biol., vol. 40, no. 11, pp. 2707-2714, Nov. 2014.

[17] G. E. Trahey, J. W. Allison, and O. T. Von Ramm, "Angle independent ultrasonic detection of blood flow," IEEE Trans. Biomed. Eng., vol. BME-34, no. 12, pp. 965-967, Dec. 1987.

[18] L. N. Bohs, B. J. Geiman, M. E. Anderson, S. C. Gebhart, and G. E. Trahey, "Speckle tracking for multi-dimensional flow estimation," Ultrasonics, vol. 38, nos. 1-8, pp. 369-375, Mar. 2000.

[19] Y. Seo, T. Ishizu, and K. Aonuma, "Current status of 3-dimensional speckle tracking echocardiography: A review from our experiences," J. Cardiovascular Ultrasound, vol. 22, no. 2, pp. 49-57, 2014.

[20] S. Fadnes, S. A. Nyrnes, H. Torp, and L. Lovstakken, "Shunt flow evaluation in congenital heart disease based on two-dimensional speckle tracking," Ultrasound Med. Biol., vol. 40, no. 10, pp. 2379-2391, 2014.

[21] H. Takahashi, H. Hasegawa, and H. Kanai, "Echo speckle imaging of blood particles with high-frame-rate echocardiography," Jpn. J. Appl. Phys., vol. 53, no. 7S, p. 07KF08, 2014.

[22] H. Gao, B. Heyde, and J. D'hooge, "3D intra-cardiac flow estimation using speckle tracking: A feasibility study in synthetic ultrasound data," in Proc. IEEE Int. Ultrason. Symp. (IUS), Jul. 2013, pp. 68-71.

[23] H. B. Kim, J. R. Hertzberg, and R. Shandas, "Development and validation of echo PIV," Experim. Fluids, vol. 36, no. 3, pp. 455-462, Mar. 2004.

[24] G.-R. Hong, M. Kim, G. Pedrizzetti, and M. A. Vannan, "Current clinical application of intracardiac flow analysis using echocardiography," J. Cardiovascular Ultrasound, vol. 21, no. 4, pp. 155-162, Dec. 2013.

[25] M. Tanter and M. Fink, "Ultrafast imaging in biomedical ultrasound," IEEE Trans. Ultrason., Ferroelectr, Freq. Control, vol. 61, no. 1, pp. 102-119, Jan. 2014.

[26] M. Cikes, L. Tong, G. R. Sutherland, and J. D'hooge, "Ultrafast cardiac ultrasound imaging: Technical principles, applications, and clinical benefits," JACC, Cardiovascular Imag., vol. 7, no. 8, pp. 812-823, 2014.

[27] L. Sandrin, S. Catheline, M. Tanter, X. Hennequin, and M. Fink, "Time-resolved pulsed elastography with ultrafast ultrasonic imaging," Ultrason. Imag., vol. 21, no. 4, pp. 259-272, Oct. 1999.

[28] M. Tanter, J. Bercoff, L. Sandrin, and M. Fink, "Ultrafast compound imaging for 2-D motion vector estimation: Application to transient elastography," IEEE Trans. Ultrason., Ferroelectr., Freq. Control, vol. 49, no. 10, pp. 1363-1374, Oct. 2002.

[29] S. Nikolov and J. A. Jensen, "Virtual ultrasound sources in highresolution ultrasound imaging," Proc. SPIE, vol. 4687, pp. 395-405, Apr. 2002.

[30] J. Bercoff et al., "Ultrafast compound Doppler imaging: Providing full blood flow characterization," IEEE Trans. Ultrason., Ferroelectr., Freq. Control, vol. 58, no. 1, pp. 134-147, Jan. 2011. 
[31] B.-F. Osmanski, G. Montaldo, and M. Tanter, "Out-of-plane Doppler imaging based on ultrafast plane wave imaging," IEEE Trans. Ultrason., Ferroelectr, Freq. Control, vol. 62, no. 4, pp. 625-636, Apr. 2015.

[32] L. Lovstakken, S. A. Nyrnes, B. O. Haugen, and H. Torp, "Angleindependent quantification of complex flow patterns in congenital heart disease," in Proc. IEEE Int. Ultrason. Symp., Oct. 2011, pp. 1246-1249.

[33] S. Fadnes, S. A. Nyrnes, A. Swillens, H. Torp, and L. Lovstakken, "Shunt quantification in congenital heart disease based on two-dimensional speckle tracking," in Proc. IEEE Int. Ultrason. Symp., Oct. 2012, pp. 1877-1880.

[34] C. Papadacci, M. Pernot, M. Couade, M. Fink, and M. Tanter, "High-contrast ultrafast imaging of the heart," IEEE Trans. Ultrason., Ferroelectr., Freq. Control, vol. 61, no. 2, pp. 288-301, Feb. 2014.

[35] B.-F. Osmanski, D. Maresca, E. Messas, M. Tanter, and M. Pernot, "Transthoracic ultrafast Doppler imaging of human left ventricular hemodynamic function," IEEE Trans. Ultrason., Ferroelectr., Freq. Control, vol. 61, no. 8, pp. 1268-1275, Aug. 2014.

[36] H. Takahashi, H. Hasegawa, and H. Kanai, "Intraventricular blood flow vector and streamline imaging using high frame rate cardiac ultrasound," in Proc. IEEE Int. Ultrason. Symp., Sep. 2014, pp. 341-344.

[37] A. Swillens, L. Løvstakken, J. Kips, H. Torp, and P. Segers, "Ultrasound simulation of complex flow velocity fields based on computational fluid dynamics," IEEE Trans. Ultrason., Ferroelectr., Freq. Control, vol. 56 , no. 3, pp. 546-556, Mar. 2009.

[38] A. Swillens, J. Degroote, J. Vierendeels, L. Lovstakken, and P. Segers, "A simulation environment for validating ultrasonic blood flow and vessel wall imaging based on fluid-structure interaction simulations: Ultrasonic assessment of arterial distension and wall shear rate," Med. Phys., vol. 37, no. 8, pp. 4318-4330, Aug. 2010.

[39] A. Swillens, P. Segers, H. Torp, and L. Lovstakken, "Two-dimensional blood velocity estimation with ultrasound: Speckle tracking versus crossed-beam vector Doppler based on flow simulations in a carotid bifurcation model," IEEE Trans. Ultrason., Ferroelectr., Freq. Control, vol. 57, no. 2, pp. 327-339, Feb. 2010.

[40] A. Swillens, P. Segers, and L. Lovstakken, "Two-dimensional flow imaging in the carotid bifurcation using a combined speckle tracking and phase-shift estimator: A study based on ultrasound simulations and in vivo analysis," Ultrasound Med. Biol., vol. 36, no. 10, pp. 1722-1735, Oct. 2010

[41] A. Swillens, G. De Santis, J. Degroote, L. Lovstakken, J. Vierendeels, and P. Segers, "Accuracy of carotid strain estimates from ultrasonic wall tracking: A study based on multiphysics simulations and in vivo data," IEEE Trans. Med. Imag., vol. 31, no. 1, pp. 131-139, Jan. 2012.

[42] K. V. Canneyt, A. Swillens, L. Lovstakken, L. Antiga, P. Verdonck, and P. Segers, "The accuracy of ultrasound volume flow measurements in the complex flow setting of a forearm vascular access," J. Vascular Access, vol. 14, no. 3, pp. 281-290, 2013.

[43] J. A. Jensen, "Field: A program for simulating ultrasound systems," Med. Biol. Eng. Comput., vol. 4, no. 1, pp. 351-353, 1996.

[44] J. A. Jensen and N. B. Svendsen, "Calculation of pressure fields from arbitrarily shaped, apodized, and excited ultrasound transducers," IEEE Trans. Ultrason., Ferroelectr., Freq. Control, vol. 39, no. 2, pp. 262-267, Mar. 1992.

[45] S. Annerel, J. Degroote, T. Claessens, P. Segers, P. Verdonck, and J. Vierendeels, "The upstream boundary condition influences the leaflet opening dynamics in the numerical FSI simulation of an aortic BMHV," Int. J. Numer. Methods Biomed. Eng., vol. 28, nos. 6-7, pp. 745-760, 2012.

[46] H. F. Choi, J. D'hooge, F. E. Rademakers, and P. Claus, "The influence of left-ventricular shape on end-diastolic fiber stress and strain," in Proc. Аnnu. Int. Conf. IEEE Eng. Med. Biol. Soc., Sep. 2009, pp. $2887-2890$

[47] T. P. Graham, J. M. Jarmakani, R. V. Canent, and M. N. Morrow, "Left heart volume estimation in infancy and childhood: Reevaluation of methodology and normal values," Circulation, vol. 43, no. 6 , pp. 895-904, Jun. 1971

[48] C. Kampmann et al., "Normal values of M mode echocardiographic measurements of more than 2000 healthy infants and children in central Europe," Heart, vol. 83, no. 6, pp. 667-672, Jun. 2000.

[49] M. Cantinotti, M. Scalese, S. Molinaro, B. Murzi, and C. Passino, "Limitations of current echocardiographic nomograms for left ventricular, valvular, and arterial dimensions in children: A critical review," J. Amer. Soc. Echocardiogr., vol. 25, no. 2, pp. 142-152, Feb. 2012.

[50] R. A. Hurwitz, S. Treves, and A. Kuruc, "Right ventricular and left ventricular ejection fraction in pediatric patients with normal hearts: First-pass radionuclide angiocardiography," Amer. Heart J., vol. 107, no. 4, pp. 726-732, Apr. 1984
[51] J. Donea, A. Huerta, J.-P. Ponthot, and A. Rodríguez-Ferran, "Arbitrary Lagrangian-Eulerian methods," in Encyclopedia of Computational Mechanics, E. Stein, R. De Borst, and T. J. R. Hughes, Eds. New York, NY, USA: Wiley, 2004.

[52] T. F. Mackintosh and C. H. Walker, "Blood viscosity in the newborn," Arch. Disease Childhood, vol. 48, no. 7, pp. 547-553, 1973.

[53] J. M. Thijssen, "Ultrasonic speckle formation, analysis and processing applied to tissue characterization," Pattern Recognit. Lett., vol. 24 nos. 4-5, pp. 659-675, Feb. 2003.

[54] L. Tong, H. Gao, H. F. Choi, and A. J. D'hooge, "Comparison of conventional parallel beamforming with plane wave and diverging wave imaging for cardiac applications: A simulation study," IEEE Trans. Ultrason., Ferroelectr., Freq. Control, vol. 59, no. 8, pp. 1654-1663, Aug. 2012.

[55] K. Mori, Y. Hayabuchi, Y. Kuroda, M. Nii, and T. Manabe, "Left ventricular wall motion velocities in healthy children measured by pulsed wave Doppler tissue echocardiography: Normal values and relation to age and heart rate," J. Amer. Soc. Echocardiogr., vol. 13, no. 11, pp. 1002-1011, Nov. 2000 .

[56] L. Lovstakken, S. Bjaerum, K. Kristoffersen, R. Haaverstad, and H. Torp, "Real-time adaptive clutter rejection filtering in color flow imaging using power method iterations," IEEE Trans. Ultrason., Ferroelectr., Freq. Control, vol. 53, no. 9, pp. 1597-1608, Sep. 2006.

[57] Y. M. Yoo, R. Managuli, and Y. Kim, "Adaptive clutter filtering for ultrasound color flow imaging," Ultrasound Med. Biol., vol. 29, no. 9, pp. 1311-1320, Sep. 2003.

[58] J. Degroote and J. Vierendeels, "Multi-solver algorithms for the partitioned simulation of fluid-structure interaction," Comput. Methods Appl. Mech. Eng., vol. 200, nos. 25-28, pp. 2195-2210, Jun. 2011.

[59] J. Degroote and J. Vierendeels, "Multi-level quasi-Newton coupling algorithms for the partitioned simulation of fluid-structure interaction," Comput. Methods Appl. Mech. Eng., vols. 225-228, pp. 14-27, Jun. 2012.

[60] H. Gao, N. Bijnens, D. Coisne, M. Lugiez, M. Rutten, and J. D'hooge, "2-D left ventricular flow estimation by combining speckle tracking with Navier-Stokes-based regularization: An in silico, in vitro and in vivo study," Ultrasound Med. Biol., vol. 41, no. 1, pp. 99-113, Jan. 2015.

[61] M. Arigovindan, M. Suhling, C. Jansen, P. Hunziker, and M. Unser, "Full motion and flow field recovery from echo Doppler data," IEEE Trans. Med. Imag., vol. 26, no. 1, pp. 31-45, Jan. 2007.

[62] T. Uejima et al., "A new echocardiographic method for identifying vortex flow in the left ventricle: Numerical validation," Ultrasound Med. Biol., vol. 36, no. 5, pp. 772-788, May 2010.

[63] D. Garcia et al., "Two-dimensional intraventricular flow mapping by digital processing conventional color-Doppler echocardiography images," IEEE Trans. Med. Imag., vol. 29, no. 10, pp. 1701-1713, Oct. 2010.

[64] F. Mehregan et al., "Doppler vortography: A color Doppler approach to quantification of intraventricular blood flow vortices," Ultrasound Med. Biol., vol. 40, no. 1, pp. 210-221, Jan. 2014.

[65] A. Gomez, K. Pushparajah, J. M. Simpson, D. Giese, T. Schaeffter, and G. Penney, "A sensitivity analysis on 3D velocity reconstruction from multiple registered echo Doppler views," Med. Image Anal., vol. 17, no. 6, pp. 616-631, Aug. 2013.

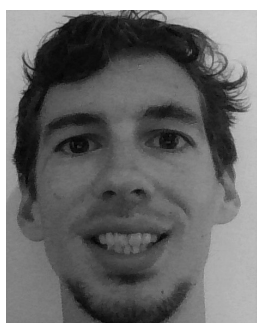

Joris Van Cauwenberge (S'15) was born in Ghent, Belgium, in 1990. He received the master's degree in biomedical engineering from Ghent University, Ghent, in 2014.

$\mathrm{He}$ is currently with the Institute Biomedical Technology, Ghent University. His current research interests include multiphysics modeling for cardiovascular ultrasound, in particular multidimensional flow imaging. 


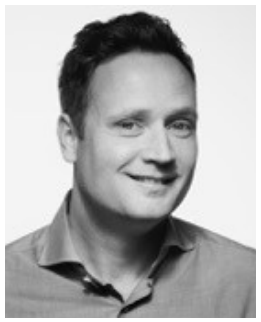

Lasse Løvstakken (S'05-M'07) received the M.Sc. degree in engineering cybernetics and the Ph.D. degree in medical ultrasound imaging technology from the Norwegian University of Science and Technology (NTNU), Trondheim, Norway.

$\mathrm{He}$ is currently a Professor with the Department of Circulation and Medical Imaging, NTNU. His current research interests include signal and image processing applied in medical ultrasound imaging for improved image formation and for the estimation of tissue and blood velocities, and cardiovascular disease with an emphasis on methods for improved estimation of blood velocities in the heart and vessels.

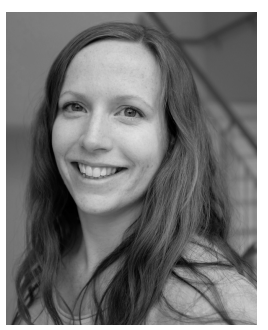

Solveig Fadnes (A'15) was born in Ålesund, Norway, in 1986. She received the M.Sc. degree in applied physics and the Ph.D. degree in medical technology from the Norwegian University of Science and Technology (NTNU), Trondheim, Norway, in 2010 and 2013, respectively.

She is currently a Post-Doctoral Fellow with the Department of Circulation and Medical Imaging, NTNU. Her current research interests include signal processing and blood flow imaging in diagnostic ultrasound.



Alfonso Rodriguez-Molares was born in $\mathrm{La}$ Coruna, Spain, in 1980. He received the M.Sc. degree in telecommunications and the Ph.D. degree in signal processing and communication from the University of Vigo, Vigo, Spain, in 2006 and 2011, respectively.

$\mathrm{He}$ is currently a Lecturer and a Researcher with the Department of Circulation and Medical Imaging, Norwegian University of Science and Technology, Trondheim, Norway. He has authored over ten papers in international peer-reviewed journals. He holds two patents in the field of acoustics and ultrasound. His current research interests include ultrasound imaging, adaptive beamforming, acoustic propagation in heterogeneous media, and shear wave elasticity.



Jan Vierendeels was born in 1968. He received the M.Sc. degree in electromechanical engineering, the M.Sc. degree in aeronautical and astronautical engineering, the M.Sc. degree in biomedical engineering, and the Ph.D. degree in electromechanical engineering from Ghent University, Ghent, Belgium, in 1991, 1993, 1996, and 1998, respectively.

Since 2008, he has been a Full Professor of Fluid Mechanics with the Department of Flow, Heat and Combustion Mechanics, Ghent University. His current research interests include algorithm development for fluid-structure interaction and other coupled problems with partitioned solvers, applications of fluid-structure interaction (fuel rod bundles, biomedical flows, slamming of flexible structures on a water surface, and water wave impact on flexible structures), development of computational fluid dynamics algorithms, grid generation algorithms, and applications of fluid mechanics in general.



Patrick Segers was born in Ninove, Belgium, in 1968. He received the Civil Engineer degree and the Ph.D. degree in applied sciences from Ghent University, Ghent, Belgium, in 1991 and 1997, respectively.

Since 2003, he has been a Full-Time Research Professor with the Institute Biomedical Technology, Ghent University. His current research interests include early noninvasive diagnosis of cardiovascular disease, with an emphasis on large artery function and stiffness, researching this through experimental and mathematical modeling and simulation, and in vivo research.

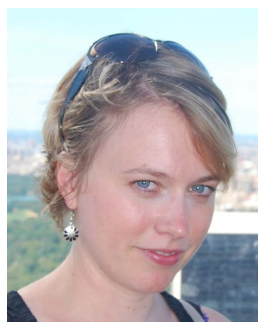

Abigail Swillens was born in Ghent, Belgium, in 1983. She received the master's degree in engineering physics from Ghent University, Ghent, in 2006, and a joint Ph.D. degree in biomedical engineering from Ghent University and the Norwegian University of Science and Technology, Trondheim, Norway, in 2010

Her current research interests include multiphysics modeling for the development of cardiovascular ultrasound imaging. 